\title{
Eine Mikromethode zur Bestimmung von Diphenylhydantoin (Phenhydan) im Blut durch Flüssigkeits-Chromatographie mit hohen Eingangsdrucken
}

\author{
Von Gisela Gauchel, F. D. Gauchel und L. Birkofer
}

\author{
Universitätskinderklinik Diisseldorf (Direktor: Prof. Dr. G. A. von Harnack) und Institut fïr Organische Chemie \\ (Direktor: Prof. Dr. L. Birkofer) der Universität Düsseldorf
}

(Eingegangen am 23. August/11. Oktober 1972)

Es wird ein flüssigkeitschromatographisches Verfahren mit hohen Eingangsdrucken zur Analyse von Diphenylhydantoin in kleinen Blutproben beschrieben. Nach Oxidation von Diphenylhydantoin wird das entstehende Benzophenon mit $i$-Octan ausgeschüttelt und durch Adsorptionschromatographie mit Naphthalin als innerem Standard bestimmt. Geht man von $50 \mu 1$ Serum aus, so beträgt die minimale quantitativ bestimmbare Diphenylhydantoin-Konzentration $1 \mathrm{mg} / 1$ und die relative Standardabweichung $6 \%$ im therapeutischen Bereich.

\section{A micromethod for the determination of diphenylhydantoin(Phenbydan) in blood by bigh speed liquid chromatography}

A micromethod for the determination of diphenylhydantoin in small blood samples by high speed liquid chromatography is reported. Diphenylhydantoin is converted to diphenyl ketone, which is extracted into $i$-Octane and determined by adsorption chromatography on silica gel, using naphthalene as an internal standard. The minimal quantitatively detectable concentration of diphenylhydantoin is $1 \mathrm{mg} / \mathrm{l}$, using a $50 \mu \mathrm{l}$ sample of serum, with a relative standard deviation at therapeutic plasma levels of $6 \%$.

Abgesehen von seiner Verwendung in der Kardiologie ist Diphenylhydantoin ein häufig zur Behandlung von Anfallsleiden benutztes Medikament. Während Konzentration im Blut, klinischer Effekt und Intoxikation eng korrelieren, ist der Zusammenhang dieser Größen bzw. Symptome mit der auf Körpergewicht und Tag bezogenen Diphenylhydantoin-Dosis großen Schwankungen unterworfen. Die Ursachen hoher oder niedriger Toleranzen sind nur teilweise bekannt, angeborene Defekte des hydroxylierenden mikrosomalen Systems der Leber, erworbene Leberfunktionsstörungen und urämische Stoffwechsellagen werden als Faktoren diskutiert. Auf entsprechende Literaturübersichten sei verwiesen $(1-3)$.

Diphenylhydantoin-Spiegel können mit Hilfe spektrophotometrischer (4-8), dünnschichtchromatographischer (9-11) und gaschromatographischer $\mathrm{Me}-$ thoden $(8,12-23)$ analysiert werden. Diese Bestimmungen erfordern in der Regel größere Blutvolumina oder zusätzliche Trennschritte.

Im folgenden wird ein einfaches flüssigkeitschromatographisches Verfahren mit hohen Eingangsdrucken beschrieben, das die quantitative Bestimmung von Diphenylhydantoin in $50 \mu \mathrm{l}$ Serum ermöglicht. Zur flüssigkeitschromatographischen Analyse therapeutischer Diphenylhydantoin-Konzentrationen in kleinen Blutvolumina mit Hilfe von UV-Detektoren ist der molare Extinktionskoeffizient dieser Substanz zu gering. Aus diesem Grunde eignet sich die von ANDERS et al. (24) angegebene flüssigkeitschromatographische Trennung von Diphenylhydantoin an Ionenaus- tauschern schlecht für Untersuchungen der Konzentration im Blut.

Wir modifizierten daher ein von WALLACE (25) angegebenes Bestimmungsverfahren, bei dem Diphenylhydantoin durch Oxidation mit Kaliumpermanganat im alkalischen Milieu zu Benzophenon oxidiert wird. Durch anschließende Extraktion und Adsorptionschromatographie an Kieselgel bei hohen Eingangsdrucken erhielten wir ein Mikroverfahren, das sich inzwischen im klinisch-chemischen Routinebetrieb bewährt hat. Über Ergebnisse dieser Untersuchungen bei anfallskranken Kindern wird demnächst an anderer Stelle berichtet.

\section{Methodik}

\section{Reagenzien}

Diphenylhydantoin-Reinsubstanz und Phenhydan zur i. v.-Injektion wurden uns freundlicherweise von Fa. Desitin-Werk Carl Klinke GmbH zur Verfügung gestellt. Alle anderen Reagenzien wurden von Fa. Merck AG, Darmstadt, bezogen.

\section{Geräte}

Hochdruckchromatograph UFC 1000, Fa. Hupe \& Busch, Grötzingen (Karlsruhe).

UV-Absorptions-Detektor 254 nm LDC 1205, Fa. Hupe \& Busch (Doppelstrahlgerät, Meßzellen: $1 \mathrm{~mm}$ Durchmesser, $10 \mathrm{~mm}$ Lichtweg, $8 \mu \mathrm{l}$ Volumen, linearer Bercich bis 0,64 Extinktionseinheiten, minimale nachweisbare Extinktion (gleich Rauschen) 0,0001 Extinktionseinheiten.

Kompensationsschreiber Typ 19, Fa. Honeywell.

Mikrolitcrspritze Typ B, Fa. Scicntific Glass Engineering PTY. Zentrifuge Christ Junior Typ UJ2, Fa. Christ, Osterode.

Whirlmix, Fa. Cenco Instrumenten MIJ N. V., Breda, Niederlande. 
Eppendorf Mikrolitersystem, Fa. Eppendorf, Hamburg. Spektralphotometer PM 4, Fa. Zeiss, Oberkochen. Fiolax Reagenzgläser $8 \times 70 \mathrm{~mm}, \mathrm{Fa}$. Schott, Mainz. Kapillaren $2 \mathrm{~mm}$ Durchmesser.

Parafilm M, American CAN-Co.

OTT-Kompensationsplanimeter Nr. 19 für $4 \mathrm{~mm}$ Meßeinheit, Fa. Bender und Hobein, München.

\section{Flüssigkeitschromatographische Analyse \\ Herstellung von Stamm- und Eicblösungen}

Stammlösung $1 \mathrm{~g} / 1$ Diphenylhydantoin: $10 \mathrm{mg}$ Diphenylhydantoin werden in $10 \mathrm{ml} \mathrm{NaOH} 0,1 \mathrm{~mol} / 1$, gelöst.

Eichlösungen 0,$1 ; 0,5 ; 1,0 ; 5,0 ; 10,0 ; 20,0 ; 50,0$ und $80,0 \mathrm{mg} / \mathrm{l}$ Diphenylhydantoin: $1,0-800 \mu \mathrm{l}$ Stammlösung werden jeweils mit Diphenylhydantoin-freiem Serum ad 10,0 ml verdünnt.

Arbeitsvorscbrift $A$ zur Bestimmung von Diphenylbydantoinkonzentrationen iber $1 \mathrm{mg} / \mathrm{l}$ in $50 \mu \mathrm{l}$ Serum

In ein $8 \times 70 \mathrm{~mm}$ Reagenzglas werden $125 \mathrm{mg} \mathrm{KMnO}_{4}, 0,4 \mathrm{ml}$ $\mathrm{NaOH} 10 \mathrm{~mol} / 1$ und $0,35 \mathrm{ml} \mathrm{NaCl}$-Lösung $0,154 \mathrm{~mol} / \mathrm{l}$ gegeben und am Whirlmix gemischt. Anschließend werden in das Reaktionsgemisch $50 \mu \mathrm{l}$ Serum bzw. Eichlösung und $50 \mu \mathrm{l} i$-Octan mit $1,0 \mu \mathrm{g}$ Naphthalin (20 mg/l Naphthalin in $i$-Octan) als innerem Standard pipettiert. Nach Verschluß mit einem Korkstopfen wird erneut am Whirlmix durchmischt.

Nach 40min Erhitzen im Metall-Thermostaten (Fa. Eppendorf) auf $95^{\circ} \mathrm{C}$, kräftigem Durchmischen, Abkühlen auf Raumtemperatur und Zentrifugation wird die organische Phase mit Hilfe einer Marburg-Pipette in eine Kapillare überführt.

Zum leichteren Abpipettieren der organischen Phase kann das Reaktionsgefäß mit $1 \mathrm{ml}$ dest. Wasser vor der Zentrifugation aufgefüllt werden. Die Probe kann in der mit Parafilm abgedichteten Kapillare mehrere Tage im Kühlschrank aufbewahrt werden.

Arbeitsvorscbrift $B$ zur Bestimmung von Diphenylbydantoinkonzentrationen unter $1 \mathrm{mg} / 1$ in $1,0 \mathrm{ml}$ Serum

$1,0 \mathrm{ml}$ Serum bzw. Eichlösung wird in einem $10 \mathrm{ml}$ Zentrifugerglas mit $0,25 \mathrm{ml} \mathrm{NaH} \mathrm{PO}_{4} 3 \mathrm{~mol} / \mathrm{l}$, versetzt, zweimal mit je $6 \mathrm{ml}$ mit Wasser gesättigtem Äther am Whirlmix kräftig gemischt und zentrifugiert. Die ätherische Phase wird in ein zweites Zentrifugenglas überführt und mit $0,5 \mathrm{ml} \mathrm{NaOH} 5 \mathrm{~mol} / \mathrm{l}$ ausgeschüttelt. Nach Zentrifugation wird die ätherische Phase verworfen. In ein $8 \times 70 \mathrm{~mm}$ Reagenzglas werden etwa $5 \mathrm{mg} \mathrm{KMnO}_{4}, 0,4 \mathrm{ml}$ der wäßr. Phase und $50 \mu \mathrm{l} i$-Octan mit 1,0 $\mu \mathrm{g}$ Naphthalin als innerem Standard gegeben. Die weitere Aufarbeitung erfolgt wie in Arbeitsvorschrift A.

Arbeitsvorscbrift $C$ zur Bestimmung von Dipbenjlbj'dantoinkonzentrationen in eiveißarmen Flissigkeiten (z. B. Urin, Speicbel, Ultrafiltrat)

In ein $8 \times 70 \mathrm{~mm}$ Reagenzglas werden $150 \mathrm{mg} \mathrm{KMnO}_{4}, 0,4 \mathrm{ml}$ $\mathrm{NaOH} 10 \mathrm{~mol} / \mathrm{l}, 0,4 \mathrm{ml}$ Probelösung und $50 \mu \mathrm{l} i$-Octan mit $1,0 \mu \mathrm{g}$ Naphthalin als innerem Standard gegeben und nach Arbeitsvorschrift $A$ aufgearbeitet.

\section{Präparation der Trennsäulen}

a) Reinigung der Stahlsäulen durch sukzessives Waschen mit Chloroform, Aceton, dest. Wasser, $\mathrm{HNO}_{3} / \mathrm{H}_{2} \mathrm{O}$ (Volumina $11+91) \mathrm{H}_{3} \mathrm{PO}_{4} / \mathrm{H}_{2} \mathrm{O}$ (Volumina $5 \mathrm{l}+51$ ), dest. Wasser, Aceton, Chloroform

b) Verschluß eines Säulenendes mit Quarzwolle

c) Stopfen der Säule durch portionsweises Einfüllen der stationären Phase und vorsichtiges Klopfen.

\section{Fliissigkeitschromatographiscbe Bedingungen ',}

Stahlsäule $1000 \times 2 \mathrm{~mm}, \quad 25$ at, Durchflußgeschwindigkeit $0,47 \mathrm{ml} / \mathrm{min}$, Elutionsmittel: $i$-Octan/i-Propanol (Volumina $991+11$ ), stationäre Phase: Merckosorb SI $6030 \mu \mathrm{m}$.

Probenvolumen: $5 \mu \mathrm{l}$ der organischen Phase (Mikroliterspritze).

\section{Ergebnisse und Diskussion}

Die in der Literatur angegebenen Bedingungen zur Oxidation von Diphenylhydantoin erfordern eine vorherige Extraktion aus dem Serum. Durch Variation der Reaktionstemperatur zwischen 37 und $95^{\circ} \mathrm{C}$, der Reaktionsdauer zwischen 10 und $240 \mathrm{~min}$, der Kaliumpermanganat-Einwaage zwischen 25 und $200 \mathrm{mg}$, des Serumvolumens zwischen 50 und $100 \mu \mathrm{l}$ und des Gesamtvolumens zwischen 250 und $850 \mu 1$ wurden die oben genannten Reaktionsbedingungen gefunden mit einer Ausbeute von $90 \%$ über alle Schritte.

Die Bestimmung von Diphenylhydantoin im Serum nach Enteiweißung mit Uranylacetat oder Zinkhydroxid ergab zu geringe Analysenwerte, wahrscheinlich infolge Adsorption von Diphenylhydantoin am Niederschlag bzw. zu geringer Löslichkeit des Pharmakons in saurer Lösung. Längerer Konțakt der organischen Lösungsmittel mit Plastikgefäßen ist zu vermeiden, da eluierte Weichmacher die Bestimmung stören. Die nach Beendigung der Reaktion abgetrennte organische Phase enthält abgesehen vom inneren Standard neben Benzophenon noch weitere, bei $254 \mathrm{~nm}$ absorbierende Substanzen.

Nach unseren Erfahrungen sind bei Konzentrationen im Blut unter $20 \mathrm{mg} / 1$ nur etwa $60 \%$ der Gesamtextinktion durch Benzophenon bedingt, wie vergleichende Untersuchungen mit wäßrigen DiphenylhydantoinLösungen ergaben. Mit Hilfe der Adsorptionschromatographie können störende Substanzen abgetrennt werden.

Zur Ermittlung optimaler chromatographischer Bedingungen wurde zunächst die Polarität des Elutions-

Tab. 1

Chromatographische Trennung von Naphthalin und Benzophenon bei variabler mobiler Phase $\mathrm{L}=4000 \mathrm{~mm}, \mathrm{P}=200$ at, stationäre Phase: Kieselgel $30 \mu \mathrm{m}$, Papiervorschub $10 \mathrm{~mm} / 150 \mathrm{~s}$

\begin{tabular}{|c|c|c|c|c|c|c|}
\hline $\begin{array}{c}\text { Mobile Phase } \\
\text { Volumverhältnis } \\
i \text {-Octan:i-Propanol }\end{array}$ & $t_{0}(\mathrm{~mm})$ & $t_{\mathrm{B}}^{\mathrm{t}_{\mathrm{R}}(\mathrm{mm})}$ & zophenon & $t_{R}(m m)$ & $\begin{array}{c}\mathbf{k}^{\prime} \\
\text { Naphthalin }\end{array}$ & $\mathrm{R}$. \\
\hline $100: 0$ & 49,5 & $>500$ & $>9$ & 58,5 & 0,18 & - \\
\hline $99,9: 0,1$ & 50,0 & $>500$ & $>9$ & 57,5 & 0,15 & - \\
\hline $99,5: 0,5$ & 50,0 & $>500$ & $>9$ & 56,5 & 0,13 & - \\
\hline $99,0: 1,0$ & 50,5 & 73,5 & 0,46 & 55,5 & 0,10 & 2,75 \\
\hline $90,0: 10,0$ & 51,0 & 62,5 & 0,23 & 54,0 & 0,06 & 1,48 \\
\hline
\end{tabular}


Tab. 2

Chromatographische Trennung von Naphthalin und Benzophenon bei variablem Eingangsdruck $\mathrm{L}=4000 \mathrm{~mm}$, mobile Phase: -Octan/i-Propanol (Volumina $991+1 \mathrm{l}$ ), stationäre Phase: Kieselgel $30 \mu \mathrm{m}$, Papiervorschub: $10 \mathrm{~mm} / 150 \mathrm{~s}$

\begin{tabular}{|c|c|c|c|c|c|c|c|c|c|}
\hline$P(a t)$ & $t_{0}(s)$ & $\mathrm{u}(\mathrm{mm} / \mathrm{s})$ & $t_{R}(m m)$ & $\begin{array}{c}w(\mathrm{~mm}) \\
\text { Benzophenon }\end{array}$ & $\mathbf{N}$ & $H(m m)$ & $t_{R}(\mathrm{~mm})$ & $\begin{array}{c}w(\mathrm{~mm}) \\
\text { Naphthalin }\end{array}$ & $\mathbf{R}$ \\
\hline 50 & 2550 & 1,57 & 249 & 20,5 & 2365 & 1,70 & 187 & 14,5 & 3,54 \\
\hline 100 & 1358 & 2,92 & 135 & 12,5 & 1865 & 2,07 & 101,5 & 8,5 & 3,19 \\
\hline 150 & 948 & 4,22 & 93 & 9,0 & 1710 & 2,34 & 70 & 6,5 & 2,97 \\
\hline 200 & 755 & 5,30 & 73,5 & 7,5 & 1540 & 2,60 & 55,5 & 5,5 & 2,75 \\
\hline
\end{tabular}

mittels durch. Änderung des Verhältnisses i-Octan/ i-Propanol variiert. Die dabei resultierenden Retentionszeiten und Kapazitätsfaktoren ${ }^{1}$ ) sind aus Tabelle 1 zu entnehmen. Die Bedingungen bei einer Elutionsmittelzusammensetzung $i$-Octan/i-Propanol (Volumina $991+11$ ) scheinen mit einem Kapazitätsfaktor von 0,46 günstig, da geringere Polarität des Elutionsmittels zu einem größeren Kapazitätsfaktor für Benzophenon, längeren Analysenzeiten und geringeren Peakhöhen führt. Anschließend wurden bei konstantem Kapazitätsfaktor und konstanter Säulenlänge unterschied-

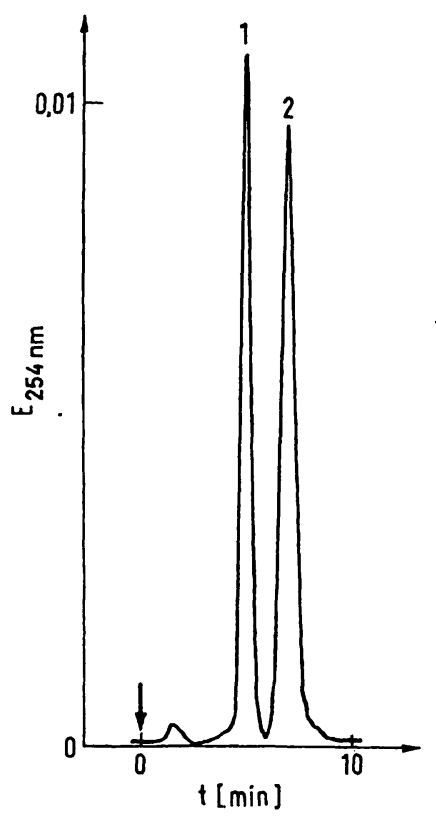

Abb. 1

Bestimmung von Diphenylhydantoin im Serum durch HochdruckFlüssigkeits-Chromatographie. Abszisse: Zeit t (min), Ordinate: Extinktion bei $254 \mathrm{~nm}$, Probe: Serum 5 min nach Injektion von $250 \mathrm{mg}$ Diphenylhydantoin (s. Abb. 2). Säule: $1000 \times 2 \mathrm{~mm}$, stationäre Phase: Merckosorb SI $6030 \mu \mathrm{m}$, mobile Phase: $i$-Octan/i-Propano (Volumina $991+1 \mathrm{l}), \mathbf{u}=0,47 \mathrm{ml} / \mathrm{min}$, innerer Standard: Naphthalin. Injiziertes Volumen: $5 \mu$ l. Peak 1 : Naphthalin, Peak 2: Benzophenon

1) Abkürzungen und Gleichungen s. Anhang. liche Eingangsdrucke eingestellt. Das Ergebnis eines Optimierungsexperimentes ist in Tabelle 2 dargestellt. Anhand bekannter physikalischer Beziehungen (26-28) läßt sich aufgrund der in Tabelle 2 crmittelten Daten eine ausreichende Trennung von Naphthalin und Benzophenon $(R=1,61)$ bei $P=25$ at, $L=1 \mathrm{~m}$ und $t_{R}=31 \mathrm{~s}$ vorbersagen. Das Experiment ergab $R=$ 1,66 bei $t_{R}=28 \mathrm{~s}$. Sobald der Benzophenonpeak die Null-Linie erreicht hat, kann die nächste Probe chromatographiert werden. Abbildung 1 zeigt ein typisches Chromatogramm.

Die Peakflächen wurden planimetrisch von Hand ausgemessen. Eichkurven wurden durch Bestimmung des Peakflächenverhältnisses Benzophenon/Naphthalin bei wechselnden Gewichtsverhältnissen angefertigt. Sie sind im untersuchten Bereich von $0,1-80 \mathrm{mg} / \mathrm{l} \mathrm{Di-}$ phenylhydantoin linear. Die relative Standardabweichung beträgt $6 \%$ für $10 \mathrm{mg} / 1$ Diphenylhydantoin. Die unteren nachweisbaren Konzentrationen sind für Arbeitsvorschrift A $1 \mathrm{mg} / \mathrm{l}$, für Arbeitsvorschrift B und C $0,1 \mathrm{mg} / 1 \mathrm{mit}$ einer relativen Standardabweichung von $16 \%$ für Arbeitsvorschrift $B$.

Als Beispiel für die Leistungsfähigkeit der Methode seien in Abbildung 2 die Ergebnisse eines Diphenylhydantoin-Belastungstests angegeben. In diesem Fall weicht der Konzentrationsverlauf von den durch A. J. Hantley (29) beschriebenen Ergebnissen ab, der eine zweigipflige Kurve fand.

Die Hochdruck-Flüssigkeits-Chromatographie sollte als Alternative zur Gaschromatographie bei der Analyse polarer, relativ schwer verdampfbarer Substanzen in Erwägung gezogen werden $(30,31)$. Sind, wie das bei klinisch-pharmakologischen Blutspiegeluntersuchungen häufig der Fall sein dürfte, nur eine geringe $\mathrm{Zahl}$ von Substanzen in der gleichen Probe zu analysieren, so wird angesichts der großen $\mathrm{Zahl}$ variabler Parameter eine flüssigkeitschromatographische Trennung in der Regel möglich sein. Die quantitativen Nachweis-

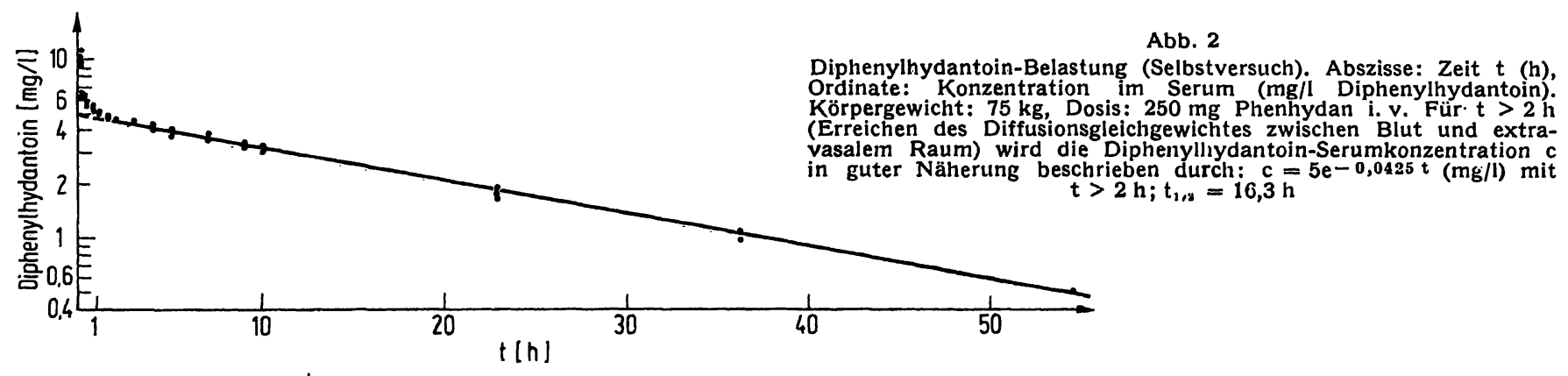


grenzen der getrennten Substanzen hängen von" deren Eigenschaften im Verhältnis zum Detektorsystem ab. Bei spektrophotometrischen Detektoren ist die Nachweisgrenze durch den molaren Extinktionskoeffzienten, die bei der Trennung erreichte Peakform und die instrumentell erreichbare Verstärkung bzw. Rauschhöhe gegeben. Bei der oben beschriebenen Meßzelle sollte die Probelösung in bezug auf jede der quantitativ " zu erfassenden Substanzen bei der zur Messung benutzten Wellenlänge eine Extinktion von mindestens 0,2 erwarten lassen. Die Nachweisgrenze ist bei gegebenem Detektor und molarem Extinktionskoeffizienten letztlich abhängig von der Verdünnung, die die Substanz bei der Passage durch die Säule erfährt. $\mathrm{Da}$ die Peakbreite variabel ist, und $z w a r$ bei der Flüssigkeitschromatographie stärker als bei der Gaschromatographie, kann die Nachweisgrenze ohne vorherige Ermittlung chromatographischer Daten nur in grober Annäherung abgeschätzt werden.

\section{Anhang}

Folgende Abkürzungen und Gleichungen werden benutzt:

L Säulenlänge

P Eingangsdruck

$t_{0}$ Durchbruchzeit

$t_{R}$ Retentionszeit

$v$ Strömungsgeschwindigkeit ( $\mathrm{ml} / \mathrm{min})$

u Strömungsgeschwindigkeit $(\grave{\mathrm{m} m} / \mathrm{s})$

w Basisbreite eines Peaks

N Zahl der theoretischen Böden

$\mathrm{H}$ Höhe eines theoretischen Bodens

R Trennfaktor

$\mathrm{k}^{\prime}$ Kapazitätsfaktor

(1) $k^{\prime}=\left(t_{R} / t_{0}\right)-1$

(2) $\mathrm{u}=\mathrm{L} / \mathrm{t}_{0}$

(3) $N=16\left(t_{R}^{2} / w^{2}\right)$

(4) $\mathrm{H}=\mathrm{L} / \mathrm{N}$

(5) $\mathrm{R}=2\left(\mathrm{t}_{\mathrm{R} 2}-t_{\mathrm{R} 1}\right) /\left(\mathrm{w}_{1}+\mathrm{w}_{2}\right)$

(6) $\mathrm{n}=\Delta \log \mathrm{H} / \Delta \log \mathrm{u}$

(7) $t_{\mathrm{R} 2} / \mathrm{t}_{\mathrm{R} 1}=\left(\mathrm{L}_{2} / \mathrm{L}_{1}\right)^{2}$

(8) $\mathrm{L}_{2} / \mathrm{I}_{1}=\left(\mathrm{R}_{2} / \mathrm{R}_{1}\right)^{2 /(1+n)}$

Gleichungen $6-8$ gelten nur für $\mathrm{k}^{\prime}=$ const.

\section{Literatur}

1. ReMmer, H. (1969), Deut. Med. Wochenschr. 94, 1265-1272. 2. Kutt, H. \& McDoweld, F. (1968), J. Amer. Med. Ass. 203 , 167-170. - 3. Garretson, L. K. (1972), Ped. Clin. N. Amer. 19, 179-191. - 4. Dill, W. A., Kazenko, A., Wolf, L. \& Glazko, A. J. (1956), J. Pharmacol. Exp. Ther. 118, 270-279. 5. PlaA, G. L. \& Hine, C. H. (1956), J. Lab. Clin. Med. 47, 649-657. - 6. Svensmark, O. \& KRISTEnSEN, P. (1963); J. Lab. Clin. Med. 61, 501-507. - 7. SAukkonen, H. \& Levonen, E. J. (1969), Scand. J. Clin. Lab. Invest. 24 Suppl. 110, 125. - 8 Berlin, A., Agurell, S., Borga, O., Lund, L. \& Sjöquist, F. (1972), Scand. J. Clin. Lab. Invest. 29, 281-287. - 9. Olesen, O. V. (1965), Acta Pharmacol. Toxicol. 23, 41-48. - 10. Hansen, J. M., Kristensen, M. \& Skovsted, L. (1968), Epilepsia 9, 17-22. - 11. Hetrand, L. B., Knowlton, D. A. \& Couri, D. (1972), Clin. Chim. Acta 36, 473-478. - 12. SandBerg, D. H. Resnik, G. L. \& BaCALlo, C. Z. (1968), Anal. Chem. 40, 763-738. 13. Grimmer, G., Jacob, J. \& SChäfer, H. (1969), Arzneim.Forsch. 19, 1287-1290. - 14. SABIH, K. \& SABIH, K. (1969), Anal. Chem. 41, 1452-1454. - 15. BAYLIS, E. M., FRY, D. E. \& Marks, V. (1970), Clin. Chim. Acta 30, 93-103. - 16. Chang,
T. \& Glazko, A. J. (1970), J. Lab. Clin. Med. 75, 145-155. 17. Evenson, M. A., Jones, P. \& Darcey, B. (1965), Clin. Chem. 16, 107-110. - 18. Kupferberg, H. J. (1970), Clin. Chim. Acta 29, 283-288. - 19. MACGeE, J. (1971), Anal. Chem. 42, 421-422. - 20. Meter, J. C. van, BuckMaster, H. S. \& Shelly L. L. (1970), Clin. Chem. 16, 135-138. - 21. Larsen, N. E. (1971), Med. Lab. Technol. 28, 377-381. - 22. MErreR, J. A. W. (1972), Epilepsia 12, 341-352. - 23. Toseland, P. A., GRove, J. \& Berry, D. J. (1972), Clin. Chim. Acta 38, 321-328. 24. Anders, M. W. \& Latorre, J. P. (1970), Anal. Chem. 42, 1430-1432. - 25. Wallace, J. E. (1969), Clin. Chem. 15 323-330. - 26. KrRkLAND, J. J. (1971), Modern Práctice of Liquid Chromatography, 1. Aufl., Wiley-Interscience New York, London, Sidney, Toronto. - 27. SNYDER, L. R. (1972), J. Chromatogr. 10, 200-213. - 28. SNYDER, L. R. (1972), J. Chromatogr. 10, 369-379. - 29. HANDLEY, A. J. (1970), Brit. Med. J. 3, 203-204. - 30. Gauchel, F. D., GaUched, G., Beyermann, K. \& ZAHN, R. K. (1972), Fresenius' Z. Anal. Chem. 259, 177 bis 183. - 31. Gaucher, G., Gauchèi, F. D., BexermanN, K. \& ZAHN, R. K. (1972), Fresenius' Z. Anal. Chem. 259, 183-187.

Dr. Dr. F. D. Gauchel Universitätskinderklinik 4 Düsseldorf 1 Moorenstr. 5 